\title{
Chemical Composition of the Pole-On Be Star HD 120991
}

\author{
Y. Frémat ${ }^{1}$, J. Zorec ${ }^{2}$, and A.-M. Hubert ${ }^{1}$ \\ 1 Observatoire de Paris, Section d'Astrophysique de Meudon, GEPI, \\ FRE K 2459, 5 Place Jules Janssen, 92195 Meudon CEDEX, France \\ 2 Institut d'Astrophysique de Paris, CNRS, 98bis Boulevard Arago, \\ 75014 Paris, France
}

\begin{abstract}
In the present work, we propose a study performed on a pole-on Be star using its IUE and FUSE spectra that we showed only slightly affected by circumstellar emission. All our computations take into account the effects of fast rotation on the spectra (differential Doppler broadening + deformation and gravitational darkening). Using the line profiles of several well known transitions and a least squares method, we determine the abundance of light elements in the photosphere of HD 120991 and compare them to the chemical composition of the interstellar medium seen in the line of sight.
\end{abstract}

\section{Introduction}

Be stars are mostly Main Sequence B type stars, burning hydrogen into their cores through the CNO cycle. The atmospheric chemical composition of slow rotating Main Sequence B type stars are generally supposed to reflect the one of the surrounding interstellar medium (ISM). However, in fast rotating objects such as Be stars it is expected that rotation introduces a turbulent diffusion of the chemical elements through the star. Due to this mixing mechanism and due to the nuclear CNO cycle, carbon should become underabundant while the abundances of helium and nitrogen should increase proportionally (Meynet \& Maeder, 2000).

In the present work, we are dealing with the FUSE and IUE data of the pole-on Be star HD 120991. The combination of optical and FUSE (Far UV Satellite Explorer) data allows us to compare the chemical composition of the star to the one of the ISM seen through the line of sight.

\section{Adopted Methodology}

The methodology we used to determine the fundamental parameters and the interstellar and photospheric abundances is described in Frémat et al. $(2002,2003)$. It accounts for NLTE effects using the TLUSTY (Hubeny \& Lanz, 1995) computer code and for the effects of stellar flattening and gravitational darkening due to fast rotation. 


\section{Results and Discussion}

The chemical composition of both the ISM and stellar photosphere was determined fitting the FUSE and IUE spectra. The nitrogen interstellar abundance was fixed by means of the $\lambda 1134 \mathrm{~N}$ I multiplet lines. It provided us the result $[\mathrm{N} / \mathrm{H}]=-4.10$ dex. We did not paid attention to carbon as it may also be used in the ISM to form molecules and dust. The $\mathrm{C}$ II and $\mathrm{N}$ II photospheric lines detected in the FUSE and IUE spectra lead to the following relative abundances $:[\mathrm{C} / \mathrm{H}]=-3.82 \pm 0.18 ;[\mathrm{N} / \mathrm{H}]=-4.01 \pm 0.21 ;[\mathrm{C} / \mathrm{N}]=0.19 \pm 0.28$. Such a low carbon to nitrogen relative abundance could be the sign of rotation induced mixing. However, the nitrogen abundance of HD120991 and of its apparent interstellar environment are not significantly different. If rotation mixing is confirmed by optical measurements, it should probably mean that HD 120991 has moved away from its protostellar environment.
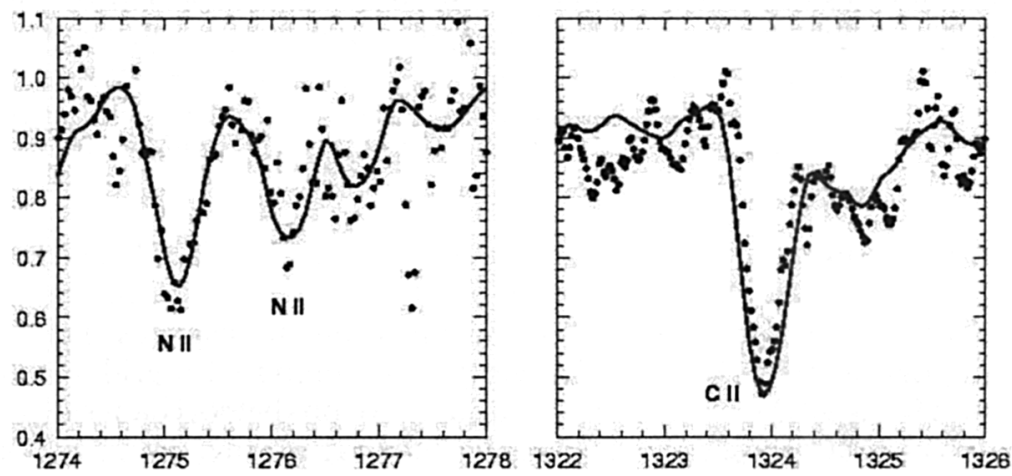

Figure 1. IUE spectrum - N II and C II observed (dots) and theoretical (line) line profiles.

Acknowledgments. This work has been supported by a Marie Curie Individual Fellowship under contract number HPMF-CT-2000-00497.

\section{References}

Frémat, Y., Zorec, J., Hubert, A.-M., Cidale, L. S., Rohrmann, R. D., Désert, J.-M., \& Ferlet, R. 2002, A\&A 385, 986

Frémat, Y., Zorec, J., Hubert, A.-M., Floquet, M., Leister, N., Levenhagen, R., Chauville, J. \& Ballereau, D. 2003, in: A. Maeder \& P. Eenens (eds.), Stellar Rotation, Proc. IAU Symp. No. 215 (San Francisco: ASP)

Hubeny, I. \& Lanz, T. 1995, ApJ 439, 875

Kurucz, R. 1994, CD-ROM No. 19.

Meynet, G. \& Maeder, A. 2000, A\&A 361, 101 